\title{
SIX PAIRWISE ORTHOGONAL LATIN SQUARES OF ORDER 69
}

\author{
L. ZHU
}

(Received 23 December 1982)

Communicated by W. D. Wallis

\begin{abstract}
In this note it is proved that a $B I B(v, k, 1)$ implies $N(v-4) \geqslant \min (N(k-2), N(k-1)-1, N(k)$ $-1)$ and that $N(69) \geqslant 6$.

1980 Mathematics subject classification (Amer. Math. Soc.): 05 B 15.
\end{abstract}

A latin square of order $v$ is a $v \times v$ matrix whose every row and every column is a permutation of a $v$-set $\Sigma$. Two latin squares of order $v$ are said to be orthogonal if when we superpose the squares every symbol in first square meets every symbol in second square exactly once. Denote by $N(v)$ the maximum number of pairwise orthogonal latin squares of order $v$. A pairwise balanced design of index unity, denoted by $P B(v ; K ; 1)$, is a design comprising a set of $v$ elements arranged in some subsets (called blocks) with block size in $K$ such that any pair of the $v$ distinct elements occur together in exactly one block. A balanced incomplete block design of index unity, denoted by $B I B(v, k, 1)$, is a $P B(v ; K ; 1)$ with $K=\{k\}$.

Using an idea of brushes due to W. D. Wallis [3], we prove in this note that a $B I B(v, k, 1)$ implies $N(v-4) \geqslant \min (N(k-2), N(k-1)-1, N(k)-1)$ and that $N(69) \geqslant 6$.

Let a brush with centre $x$ be a set of some blocks which all contain the common element $x$ but are otherwise disjoint. Two brushes are disjoint if and only if their sets of elements are disjoint. Denote by $P_{n}(k)$ the set of all integers $v$ such that

(C) 1984 Australian Mathematical Society $0263-6115 / 84 \$ A 2.00+0.00$ 
there are $n$ pairwise orthogonal latin squares of order $v$ with orthogonal subsquares of order $k$. In this way we can write $v \in P_{n}(1)$ to indicate $N(v) \geqslant n$. Similarly to Theorem 9 in [3] we have

THEOREM 1. Suppose that there is a $P B(v ; K ; 1)$ in which $B^{*}$ is a (possibly empty) distinguished set of blocks comprising a union of disjoint brushes. Suppose further that the size of any block in $B^{*}$ is in $P_{n}(1)$ and that the size of any block not in $B^{*}$ is in $P_{n+1}(1)$. Then $v \in P_{n}(k)$ where $k$ is the size of any block which is distinguished or none of whose elements is a non-central element of a brush.

Using Theorem 1, we get the following theorem which is a generalization of Theorem 13.3.4 in [2].

THEOREM 2. If there is a $B I B(v, k, 1)$, then $N(v-4) \geqslant \min (N(k-2), N(k-$ 1) $-1, N(k)-1)$.

Proof. Delete from $B I B(v, k, 1)$ four elements $y_{1}, y_{2}, y_{3}$ and $y_{4}$, such that any three of them are not in the same block. Let $l_{i j}$ be the block containing $y_{i}$ and $y_{j}$ and $l_{i j}^{*}=l_{i j} \backslash\left\{y_{i}, y_{j}\right\}$. We now get a $P B(v-4 ; k-2, k-1, k ; 1)$ with six distinguished $(k-2)$-blocks $l_{i j}^{*}, 1 \leqslant i \neq j \leqslant 4$. It is easy to see that the three groups of the six blocks $\left\{l_{12}^{*}, l_{34}^{*}\right\},\left\{l_{13}^{*}, l_{24}^{*}\right\}$ and $\left\{l_{14}^{*}, l_{23}^{*}\right\}$ are pairwise disjoint. We consider a group as a brush if its two blocks have a common element, otherwise we consider each block in a group as a brush if its two blocks have no common element. Then we get a disjoint set of brushes. Let $n=\min (N(k-2), N(k-1)$ $-1, N(k)-1)$. From Theorem 1 we have $v-4 \in P_{n}(k-2)$ and then the proof is complete.

Up until now the list of Brouwer [1] indicates that five is the last known lower bound for $N(69)$. The following theorem improves this lower bound to six.

THEOREM 3. There are six pairwise orthogonal latin squares of order 69 with orthogonal subsquares of order 7.

Proof. From [2, page 293] there is a $B I B(73,9,1)$. Since $\min (N(7)$, $N(8)-1, N(9)-1)=6($ see $[2$, Theorem 13.2.2]), we have from Theorem 2 that $69 \in P_{6}(7)$. 


\section{References}

[1] A. E. Brouwer, 'The number of mutually orthogonal latin squares, a table up to order 10,000 ,' Afd. Zuivere Wisk. Math. Centrum, Amsterdam, 1979.

[2] M. Hall, Jr., Combinatorial theory (Blaisdell, Waltham, Mass., 1967).

[3] W. D. Wallis and L. Zhu, 'Orthogonal latin squares with small subsquares,' preprint.

Department of Mathematics

Suzhou University

Suzhou

People's Republic of China 